\title{
Percutaneous Endoscopic Lumbar Approach to a Facet Joint Synovial Cyst: Case Report and Review of the Literature
}

Leonardo Giacomini ${ }^{1}$, Alexandre Bossi Todeschini ${ }^{1}$, Carlos Eduardo Vasconcelos Miranda ${ }^{1}$, Roger Neves Mathias ${ }^{1}$, Andrei Fernandes Joaquim ${ }^{2}$

${ }^{1}$ Department of Neurosurgery, Hospital 9 de Julho, ${ }^{2}$ Professor of Neurosurgery University of Campinas, Sao Paulo-SP, Brazil

Corresponding Author:

Carlos Eduardo Vasconcelos Miranda, MD it Department of Neurosurgery, Hospital 9 de Julho, Rua Paula Ney, 188 apto 81 Vila Mariana, Sao Paulo-SP \#04107-020, Brazil

Tel: $+55(11) 55313965$

Fax: $+55(11) 55313965$

E-mail: carlosevmiranda@me.com

Received: August 12, 2020

Revised: September 24, 2020

Accepted: September 25, 2020
Lumbar facet joint synovial cysts are rare lesions that may cause radiculopathy, usually it does not respond well to clinical management, oftentimes requiring surgical intervention. The percutaneous endoscopic approach may be an alternative that avoids the risk of instability. On this paper we describe the percutaneous endoscopic approach to a lumbar facet joint synovial cyst in a 78-years-old, female patient. 78-years-old female with a history of rheumatoid arthritis, which caused numerous deformities in peripheral joints. She was complaining of progressive neurogenic claudication and right-side sciatica for 6 months and a limited walking ability, the MRI revealed a $12 \mathrm{~mm}$ L4L5 right facet joint synovial cyst. The natural history of lumbar facet joint cysts is not completely understood. It has been linked in previous studies to degenerative spine diseases, such as spondylolistheses and scoliosis. There are rare publications or studies confirming the superiority of the endoscopic approach to these cysts due to its rarity. The percutaneous endoscopic approach may be an alternative. The percutaneous endoscopic approach to the lumbar. facet joins cysts is a viable approach

Key Words: Lumbar facet joint disiase, Percutaneous endoscopic approach, Synovial cysts

\section{INTRODUCTION}

The classification of spinal cysts are based in histopathology, localization and etiology. Different types of cyst such as synovial cyst, arachnoid cyst, cyst of the ligamentum flavum, discal cyst and ganglias are present in the spine". The localization can be intradural e extradural and this will determine what type of surgical access will be used. Among the different types of cyst the synovial cyst is the most commonly found").

Lumbar facet joint synovial cysts are rare lesions that may cause radiculopathy, neurogenic claudication and even cauda equina syndrome ${ }^{2,47}$. The most frequent site where it is found is anterior to the yellow ligament, postero-lateral to the dura and its contents, at the $L 4-5$ level ${ }^{4-6)}$. It does not respond well to clinical management, oftentimes requiring surgical intervention. Different approaches may be used, such as corticosteroids and local anesthetic injections, Computed Tomography (CT) guided needle aspiration, microscopic resection and, nowadays, endoscopic resection ${ }^{2-4}$. These cysts seldom disappear without intervention and the open microscopic approach may lead to spinal instability after removing part of the facet joint. The percutaneous endoscopic approach is a viable option that avoids the risk of instability.

We aim to describe the percutaneous endoscopic approach to a lumbar facet joint synovial cyst in a 78-years-old, female patient.

\section{CASE REPORT}

Our patient was a 78-years-old female with a history of rheumatoid arthritis, which caused numerous deformities in peripheral joints, and thrombophilia, which had previously precipitated four episodes of deep vein thrombosis in her lower limbs and one episode of pulmonary embolism and required continuous use of rivaroxaban.

She presented to our clinic complaining of progressive neurogenic claudication and right side sciatica for 6 months and a limited walking ability (around 50 meters). She underwent mag- 
netic resonance imaging (MRI) of the lumbar spine which revealed a $12 \mathrm{~mm}$ L4-5 right facet joint synovial cyst. This cyst caused significant spinal stenosis and it was the most likely cause of her symptoms (Fig. 1).

The patient's previous history of thrombophilia forbade discontinuing oral anticoagulant, therefore we proposed a minimally invasive percutaneous endoscopic approach. The posterior position of the cyst to the cauda equina facilitated our choice and approach.
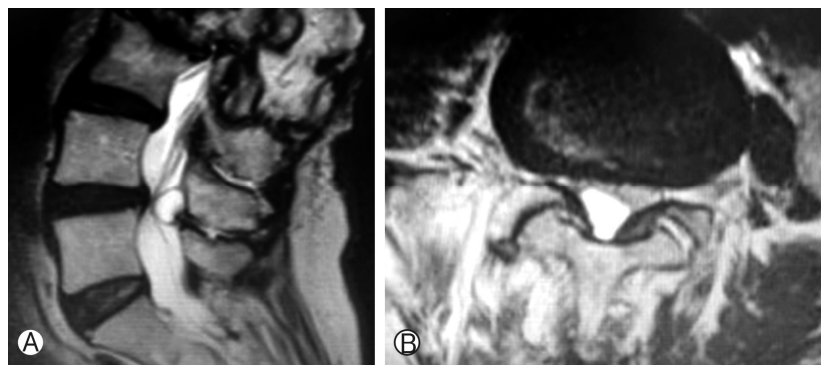

Fig. 1. T2 weighted MRI images of the lumbar spine in the sagittal (A) and axial (B) views. Note the epidural cyst adjacent to the L4L5 facet joint and the compression of the cauda equina and emerging nerve root.
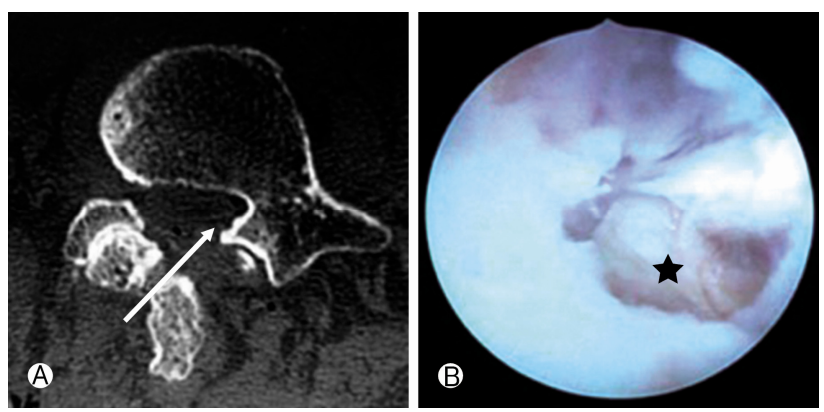

Fig. 2. We approached the posterior portion of the spinal canal through the inferior articular facet (A). Endoscopic view of the synovial cyst capsule (B), black star).
Surgery was done under general anesthesia without somatosensory evoked potentials (SSEPs). Using fluoroscopy to identify the target level and the significant landmarks, we introduced the spinal needle on the right side of the patient, $10 \mathrm{~cm}$ lateral to the midline advancing under anteroposterior fluoroscopy toward the inferior plateau of $L 4$ vertebra. Using latero-lateral view in the fluoroscopy we aimed and advanced towards in the L4's inferior articular facet-lamina transition. For endoscopic discectomies, the target(the intervertebral space and disc) is more anterior than our current case and the bony landmark used to introduce the needle is the superior articular facet of the inferior vertebra. However, to access the spinal canal (more posterior) and the structure therein, the inferior facet-lamina transition of the superior vertebra is a more suitable target.

After advancing the $18 \mathrm{G}$ needle up to the cranial portion of the inferior articular facet, a Kirshner's wire (K-wire) was introduced. An eight $\mathrm{mm}$ skin incision was done and two sequential dilators were used with the K-wire as guide. The sharp tip TOM Shidi cannula (MaxMoreSpine, Unterfõhring, Germany) was advanced $5 \mathrm{~mm}$ using a surgical mallet, followed by manual drills with a respective diameter of 4,6 , and $8 \mathrm{~mm}$ to enlarge the entrance into the facet-lamina transition created by the TOM Shidi cannula and allow the endoscope to be introduced and positioned (Fig. 2).

After introducing the endoscope, using endoscopic kerrisons rongeurs, the remaining of the articular facet was removed, allowing direct access to the cyst and resection of its capsule and contents (Fig. 3).

Post-operative course was uneventful. Immediately after surgery the sciatica resolved and neurogenic claudication improved with a complete resolution at the 12 weeks post-operative visit.

Histopathologic analysis showed the presence of synovial membrane cell with mucinous component and hemosiderin compatible with synovial cyst.

\section{DISCUSSION}

The natural history and development of lumbar facet joint
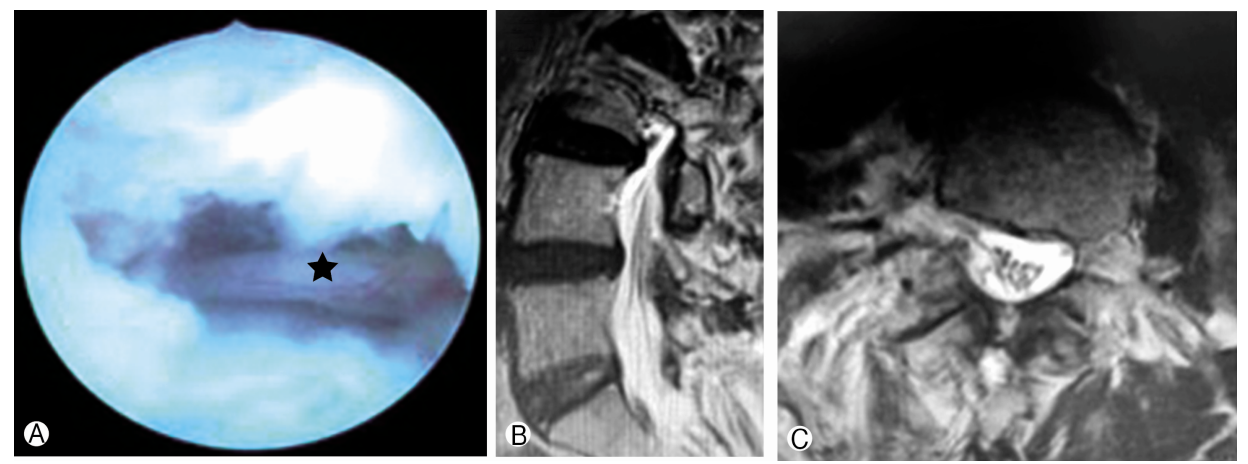

Fig. 3. (A): Final view of the procedure through the endoscope without evidence of any residual lesion and the L5 root without any compression (black star). (B) and (C): Post-operative T2-weighted MRI images in the sagittal (B) and axial (C) views showing complete resection of the cyst and decompression of the cauda equina. 
cysts is not completely understood". It has been linked in previous studies to degenerative spine diseases, such as spondylolistheses and scoliosis ${ }^{3}$. Considering this association and the fact that open microscopic approaches oftentimes lead to spinal instability, the minimally invasive treatment through an endoscopic approach may be more beneficial to patients with this lesion, avoiding instability and the need for spinal instrumentation".

Few publications have been published without showing clear superiority of the endoscopic approach to these cysts. Bruder et al. analyzed 141 cases and reported a success rate of $94 \%$ with open resection through a hemi-laminectomy. In this series, there was a 7\% recurrence and only 9\% required spinal instrumentation on follow up ${ }^{5}$. A meta-analysis by Campbel et al. reported clearly the superiority of open resection over percutaneous needle aspiration. Their study demonstrated a resolution rate of cyst compression on open resection of $90 \%$ versus a $58 \%$ on percutaneous needle aspiration ${ }^{6,9}$. Considering that lumbar facet joint cysts are more frequent in elder patients with pre-existing illnesses, which may hinder open approaches, a minimally invasive alternative with high success rates would be a welcome addition to the surgeon's armamentarium ${ }^{7,8)}$.

The percutaneous endoscopic approach may be such alternative. It allows a complete resection of the cyst and its capsule rather than just aspiration of its contents. Although there are no randomized trials comparing open and endoscopic approaches, some studies have shown the endoscopic approach to have similar success rates to open approaches ${ }^{2-4)}$. Using the percutaneous endoscopic approach, the lesion is reached through the facet joint, avoiding muscle and ligament injury incurred by the open approach. Furthermore, the open approach also requires a medial facetectomy increasing the risk of spinal instability and the need for instrumentation ${ }^{2-4)}$. A interesting point was the use of the Tomshid cannula (a non-disposable device) instead of an electric drill to access the spinal canal. This is important in relation to the cost-benefit considering the reality of Brazil.

The percutaneous endoscopic technique used for the resection of these cysts requires specialized training and experience by the surgeon to correctly identify the anatomical landmarks and avoid unintentional injury to the patient ${ }^{4}$. The current literature and data are based on small case series and reports with no randomized trials comparing both approaches.

\section{CONCLUSION}

The percutaneous endoscopic approach to the lumbar facet joins cysts is a viable approach. It should be mainly considered for patients that would benefit from shorter hospital stays, minimally invasive procedures and reducing the risk of spinal instability.

\section{REFERENCES}

1. Sun J: Classification, mechanism and surgical treatments for spinal canal cyst. Chinese Neurosurgical Journal 7:2, 2016

2. Kim HS, Singh R, Adsul N, et al: Full endoscopic treatment of unusual spontaneous degenerative epidural cyst: A case report. Surg Neurol Int 10:58, 2019

3. Wu HH, Chu L, Zhu Y, et al: Percutaneous endoscopic lumbar surgery via the transfacet approach for lumbar synovial cyst. World Neurosurgery 116:35-39, 2018

4. Oertel JM, Burkhardt BW: Endoscopic surgical treatment of lumbar synovial cyst: Detailed account of surgical technique and report of 11 consecutive patients. World Neurosurgery 103:122132, 2017

5. Bruder M, Cattani A, Gessler F, et al: Synovial cysts of the spine: long-term follow-up after surgical treatment of 141 cases in a single-center series and comprehensive literature review of 2,900 degenerative spinal cysts. Journal of Neurosurgery: Spine 27(3): 256-267, 2017

6. Campbell RJ, Mobbs RJ, Rao PJ, et al: Interventions for lumbar synovial facet joint cysts: A comparison of percutaneous, surgical decompression and Fusion Approaches. World Neurosurgery 98: 492-502, 2017

7. Boody BS, Savage JW: Evaluation and treatment of lumbar facet cysts. Journal of the American Academy of Orthopaedic Surgeons 24(12):829-842, 2016

8. Khan A M, Girardi F: Spinal lumbar synovial cysts. Diagnosis and management challenge. European Spine Journal 15(8):11761182, 2006

9. Imai K, Nakamura K, Inokuchi K, et al: Aspiration of intraspinal synovial cyst: recurrence after temporal improvement. Archives of Orthopaedic and Trauma Surgery 118(1-2):103-105, 1998 